\title{
Formation of classical crystals of dipolar particles in a helical geometry
}

\author{
J. K. Pedersen, D. V. Fedorov, A. S. Jensen, and N. T. Zinner \\ Department of Physics and Astronomy, Aarhus University, DK-8000 Aarhus C, Denmark
}

(Dated: November 5, 2018)

\begin{abstract}
We consider crystal formation of particles with dipole-dipole interactions that are confined to move in a one-dimensional helical geometry with their dipole moments oriented along the symmetry axis of the confining helix. The stable classical lowest energy configurations are found to be chain structures for a large range of pitch-to-radius ratios for relatively low density of dipoles and a moderate total number of particles. The classical normal mode spectra support the chain interpretation both through structure and the distinct degeneracies depending discretely on the number of dipoles per revolution. A larger total number of dipoles leads to a clusterization where the dipolar chains move closer to each other. This implies a change in the local density and the emergence of two length scales, one for the cluster size and one for the inter-cluster distance along the helix. Starting from three dipoles per revolution, this implies a breaking of the initial periodicity to form a cluster of two chains close together and a third chain removed from the cluster. This is driven by the competition between in-chain and out-of-chain interactions, or alternatively the side-by-side repulsion and the head-totail attraction in the system. The speed of sound propagates along the chains. It is independent of the number of chains although depending on geometry.
\end{abstract}

\section{INTRODUCTION}

A highly promising direction in cold atoms phyiscs is the experimental realization and exploration of homoand heteronuclear molecules at low temperatures [1 9]. The potential applications of such systems are wideranging within quantum simulation, information, computation, and metrology [10 13]. An important issue is the head-to-tail attraction that molecules with dipole moments have which can severely limit experimental timescales due to strong losses [5, 14]. The suggested way forward has been to confine the molecules in lowdimensional setups where the head-to-tail attraction can be controllably reduced and losses can be suppressed. An experimental confirmation of this was recently achieved with a stack of two-dimensional planes [8] and in a threedimensional optical lattice [9].

A great advantage of low-dimensional setups is that one can study the interplay of geometrical restrictions on dynamics in the presence of long-range interactions from the dipole-dipole forces. This is one of the main motivations of the present paper. In the context of condensedmatter systems in low-dimensional systems, this interplay of range and geometry is a very active area of research (see Ref. [16] for an introduction). The geometry we will consider is that of a helix where we assume that the pitch and radius can be tuned experimentally. This can be realized in different ways. Laguerre-Gaussian beams with non-zero angular momentum are one possibility (bright [18 22] or dark [23, 24]). This method has some less desirable limitations on the maximum length of the uniform helix and the variational range of the parameters [15]. These restrictions can be dealt with by working instead with light guided by an optical nanofiber. Here it was recently shown that atoms can be trapped in the evanescent field surrounding the fiber waist $25-$ 27]. A particularly interesting proposal concerns the generation of the double-helix potential known from DNA
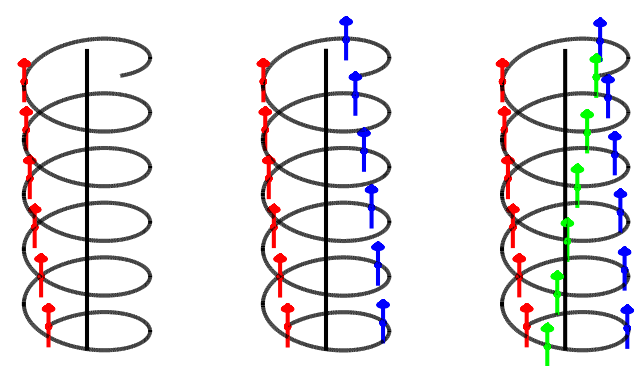

FIG. 1: Schematic drawings of chain configurations on a helix. Left is one chain, middle two chains asymmetrically located along ten helix, right is three chains. To guide the eye the dipoles in each chain have the same colours.

molecules 15.

Here we will explore the physics of a single helix setup and assume that cold molecules are trapped on the helix. A few years ago, the same geometry was discussed as a possible example of a liquid-gas transition at zero temperature 28]. Furthermore, studies of the competition between dipolar particles and the external trapping potential have been done and linear to zig-zag transition were found [29, 30]. This work builds on earlier findings of similar transitions in the case where the dipolar particles are replaced by charged ions [31, 32]. Helical structures have also been studied in spinor Bose-Einstein condensate with dipolar interactions where they can appear as spin textures [33].

In our work we will assume that the helical trap is rigid and no movement can occur away from the helix 
as done in some recent studies of the charged ions on a helix [34, 35]. This effectively means that we assume a tight trapping potential and the cost of excitations in the transverse directions will thus be much larger than the interaction energies. We illustrate the geometry in fig. 1 with different numbers of dipoles located on the helix. As we shall see they tend to form structures organized in chains depending on the density of the particles. The distance between the chains is not necessarily equidistant because the attractive and repulsive parts of the interaction strongly depend on dipole direction and distance. We are not aware of a previous discussion of the classical ground state phases in this setup with dipolar molecules.

In section II we provide energy expressions in a suitable coordinate system and focus on the simplest configurations as function of geometric helix parameters and particle number. In sections III and IV we discuss stability and normal modes, and the speed of sound in these 1D systems. Finally, section V contains a brief summary of the conclusions in addition to an outlook and perpectives related to experimental realizations.

\section{DIPOLES ON A HELIX}

We consider dipoles moving on the one dimensional geometry of a helix. An experimental realization of this geometry is possible by applying counter propagating laser beams to a tapered optical fiber [15], and trapping the dipoles in the evanescent field around the fiber. This is not strictly one dimensional, but by varying the laser power one can enter a one dimensional regime. We first specify helix parameters, dipole coodinates, potentials and energies, and second we calculate classically stable configurations.

\section{A. Classical energy}

A helix is described by two parameters, the height $h$ and the radius $R$. See fig. 2 for a schematic drawing of a helix. The radius is the distance at which the helix revolves around a fixed axis, and the height is the vertical distance between two such revolutions. The position along the helix can be described by the arc length, $s$, along the helix or equivalently by the azimuthal angle, $\phi$, around the z-axis, $\phi=s / \alpha$, where $\alpha=\left(R^{2}+\left(\frac{h}{2 \pi}\right)^{2}\right)^{1 / 2}$. We shall use the angle $\phi$ where one circle is described by $\phi \in[0,2 \pi]$, and the following by $\phi$-values increasing beyond $2 \pi$. The relation to Cartesian coordinates are given by the following transformation

$$
(x, y, z)=\left(R \sin \phi, R \cos \phi, h \frac{\phi}{2 \pi}\right) .
$$

On the helix we place $N$ identical dipoles of mass $m$ and dipole moment $\boldsymbol{d}$, all aligned along the z-axis by an external field. The dipoles are then confined to move

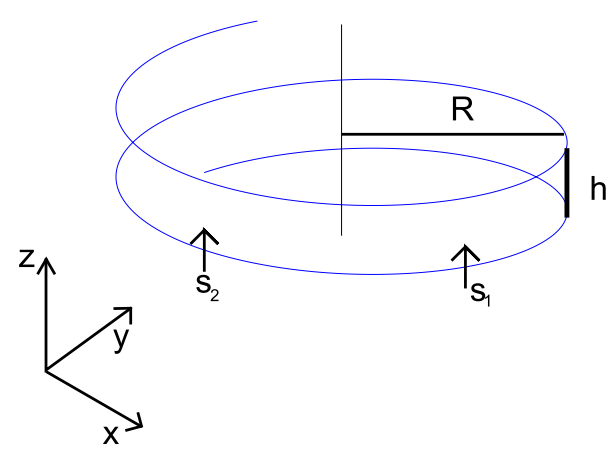

FIG. 2: Schematic drawing of a helix of radius $R$ and pitch $h$, with two dipoles, $s_{1}$ and $s_{2}$, directed along the $z$-axis.

only on the helix, but they still interact through the three dimensional Cartesian space. The potential energy, $V$, of two dipoles at position $\boldsymbol{r}_{i}$ and $\boldsymbol{r}_{j}$ is

$$
V\left(\boldsymbol{r}_{i}, \boldsymbol{r}_{j}\right)=\frac{1}{4 \pi \epsilon_{0}} \frac{1}{r^{3}}[\boldsymbol{d} \cdot \boldsymbol{d}-3(\boldsymbol{d} \cdot \hat{\boldsymbol{r}})(\boldsymbol{d} \cdot \hat{\boldsymbol{r}})],
$$

where $r=\left|\boldsymbol{r}_{i}-\boldsymbol{r}_{j}\right|$ is the distance between the dipoles, and $\hat{\boldsymbol{r}}=\left(\boldsymbol{r}_{i}-\boldsymbol{r}_{j}\right) / r$ is the unit vector in the direction connecting the two dipoles. Instead of describing the position of the dipoles by their Cartesian coordinates it turns out to be easier to use their position along the helix.

Using the transformation in Eq.(10) the potential energy of two dipoles positioned at angles $\phi_{i}$ and $\phi_{j}$ on the helix becomes

$$
\begin{aligned}
& V\left(\phi_{i}, \phi_{j}\right)=\frac{d^{2}}{4 \pi \epsilon_{0}} \\
\times & \frac{2 R^{2}\left[1-\cos \left(\phi_{i}-\phi_{j}\right)\right]-2 h^{2}\left(\left(\phi_{i}-\phi_{j}\right) /(2 \pi)\right)^{2}}{\left(2 R^{2}\left[1-\cos \left(\phi_{i}-\phi_{j}\right)\right]+h^{2}\left(\left(\phi_{i}-\phi_{j}\right) /(2 \pi)\right)^{2}\right)^{5 / 2}} .
\end{aligned}
$$

This effective potential scales as $R^{-3}$, and depends only on $h / R$ and the size of the relative angle, $|\phi|=\left|\phi_{i}-\phi_{j}\right|$, between the two dipoles.

We shall from now on use the resulting natural unit, $d^{2} /\left(4 \pi \epsilon_{0} R^{3}\right)$, for both potentials and energies. The potential from Eq.(3) in this unit is shown in fig. (3) as function of $\phi$ for intermediate values of $h$ and $R$. The repulsion for small $\phi$ when the dipoles approach each other diverge as $\phi^{-3}$. For large $\phi$ the attraction vanishes with the same power, $\phi^{-3}$.

The strongly damped oscillatory behaviour reveals several minima with depths decreasing as $\phi^{-3}$, the same power as for interacting dipoles in three dimensions. The minima are crudely located at multipla of $2 \pi$, that is the first of them around 6,12 , and 18 as seen in fig. 3. Thus, we have an effective $1 \mathrm{D}$ system with a long range dipolelike interaction.

As seen from Eq.(3) the diverging repulsion for small $\phi$ with $h=R=1$ turns into a diverging $\phi^{-3}$-attraction 


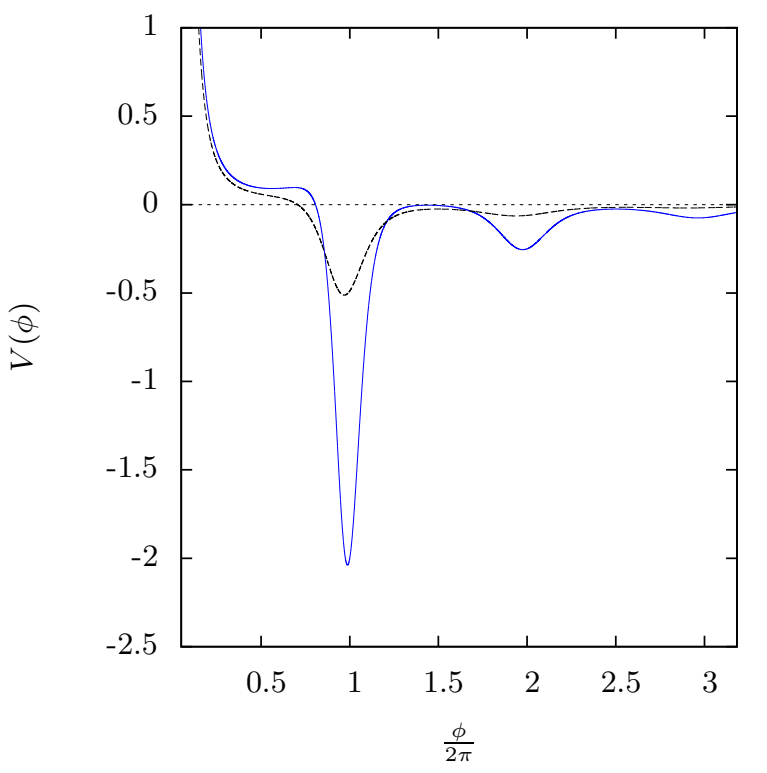

FIG. 3: The reduced potential, $4 \pi R^{3} \epsilon_{0} V / d^{2}$, of two dipoles as a function of the relative angle, $\phi=\phi_{i}-\phi_{j}$, separating the particles on the helix. The helix parameters are chosen to be $h=R$ (Blue) and $h=1.6 R$ (black).

for sufficiently large $h / R$-values, that is when $\frac{h}{R}>\pi \sqrt{2}$. The two dipoles would then prefer to be in the same point with infinite energy. In these cases other shortrange forces such as the van der Waals interaction becomes important. We want to avoid this regime where chemistry rules, and hence we limit our investigations to cases where $\frac{h}{R}<\pi \sqrt{2}$.

The total potential energy, $E$, is obtained by adding the contributions from all pairs through Eq.(3) for any set of positions given by the coordinates $\left\{\phi_{k}\right\}$, that is

$$
E\left(\left\{\phi_{k}\right\}\right)=\sum_{i<j}^{N} V\left(\phi_{i}, \phi_{j}\right) .
$$

The stable crystal configurations can then be found as minima of the energy landscape providing equilibrium values for the positions $\left\{\phi_{k}\right\}$ for any given $N$ as function of $h / R$.

\section{B. Equilibrium configurations}

Full coordinate variation of all positions imply minimization of an $N$-dimensional function. To simplify this problem we impose constraints on the positions of the dipoles. We first assume that all the dipoles are equally spaced along the helix, that is the angular distance, $\phi=\left|\phi_{i}-\phi_{j}\right|$, between neighboring particles is constant. A given total number of particles, $N$, then corresponds to varying the length of the helix. The resulting energy per dipole is shown in fig. 4 as a function

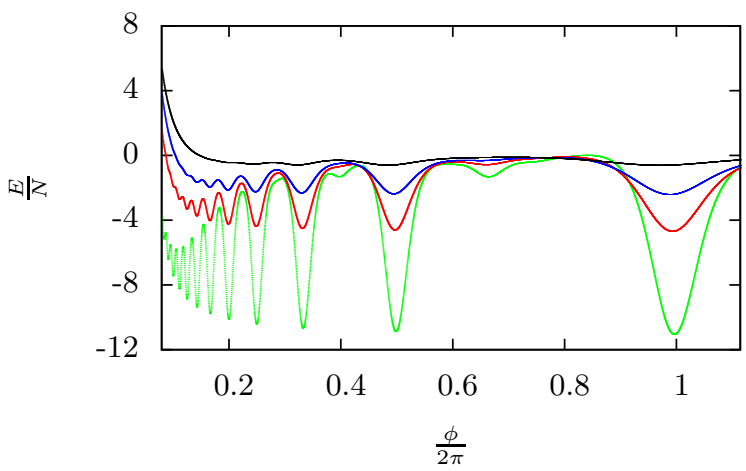

FIG. 4: The reduced energy per particle, $E / N$, in units of $d^{2} /\left(2 \pi \epsilon_{0} R^{3}\right)$ as function of the angular distance, $\phi$, between the particles. The different curves correspond to $h / R=$ 1.6, 1.0,0.8 and 0.6 with smaller well depths for decreasing $h / R$. The total particle number is $N=100$.

of $\phi$. For sufficiently large $N$ the energy per particle is independent of the length of the helix.

The oscillating functions in fig. 4 are striking. The many minima at shorter and shorter angular distance correspond to an integer number of dipoles per winding of the helix. At larger separation the oscillations continue but with cubic decrease of the depths occurring at approximately integer multipla of $2 \pi$. The depths of the minima are roughly equal for large $\phi$ while decreasing towards smaller values of $\phi$.

To interpret these observations it is useful to recall that head-to-tail vertical dipoles attract each other, while repulsion occur between horizontal dipoles connected by a vector almost perpendicular to the dipole moments. The results in fig. 3 then suggest the energy is dominated by the attraction between the dipoles in different windings, since the dominating minimum is close to $2 \pi$. The repulsion is much smaller between dipoles on the same winding, where $\phi$ differ from $2 \pi$. Furthermore, the attractions between optimally placed dipoles on the same winding decrease with decreasing values of $h / R$, see fig. 3. These conclusions emerge because the energy per dipole seems to be independent of the number of repulsions arising from dipoles on the same winding.

With this overall interpretation we can look at more detailed features. The $h / R$ dependence of the positions of the minima is very weak although visible. The minimum at the highest dipole separation, $\phi \approx 2 \pi$, is in fact slightly less than $2 \pi$. This means that each dipole in this case is not separated by exactly one winding, and thus the positions of the $N$ dipoles for such a chain rotates slowly around the axis of the helix. After about 75 layers

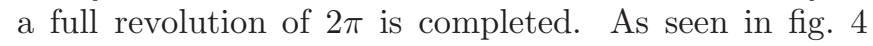
the deviation from $2 \pi$ remains essentially unchanged by modest variation of $h / R$. The second highest minimum position at around $\pi$ in fig. 4 corresponds to two dipoles per revolution. The configuration is then dipoles form- 


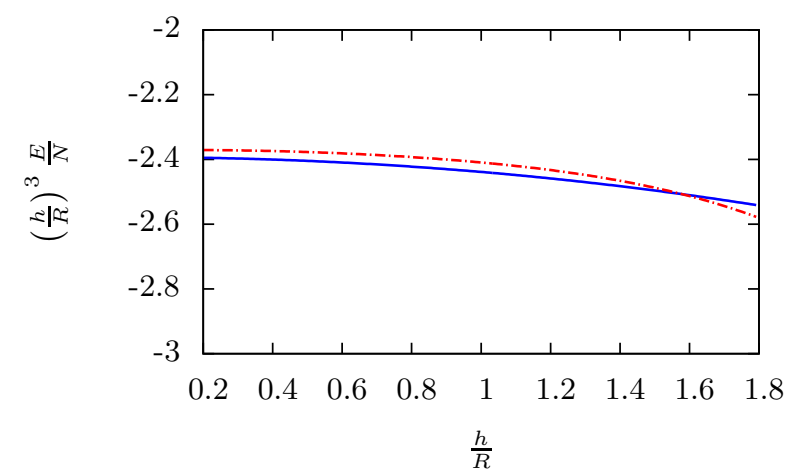

FIG. 5: Reduced energy per dipole multiplied by $(h / R)^{3}$ as a function of the ratio $\frac{h}{R}$ for one (full blue) and three (dashed red) dipoles per revolution.

ing two chains on opposite sides of the helix as illustrated in fig. 1. Closer inspection reveals that each of the two chains again slowly is rotating around the helix axis.

The energy shown in fig. 4 depends on the azimuthal angle and the ratio $h / R$ where the latter in principle can vary from 0 to $\pi \sqrt{2} \approx 4.4$, For two dipoles separated by $r$ in three dimensions the energy scales as $r^{-3}$. For two dipoles separated by one winding the three dimensional distance scales as $h$, and according to Eq.(3) the energy per dipole must scale as $h^{-3}$. If this in-chain interaction is the dominating contribution, the energy per dipole should scale in this way. This is another way to investigate the above conclusion that the attraction between dipoles in different windings dominate the total energy, even for higher dipole density.

The expected scaling is tested in fig. 5] where we show the product $\frac{E}{N}\left(\frac{h}{R}\right)^{3}$, which should be constant under these assumptions of in-chain dominance. Over the range of $h / R$ from 0.2 to 1.8 the deviations from an average constant is less than $10 \%$. This change is mostly due to the slight rotation of equilibrium angle with each revolution, i.e. dependency on both $h / R$ and the angle. The in-chain dominance would be violated by even larger values of $h$ where out-of-chain and in-chain distances become comparable. However, configurations with too large $h$-values are unstable and hence uninteresting.

The opposite limit of $h \rightarrow 0$ would also violate the distinction between particles in different chains as evident from the geometry where all particles would be on a circle and all repelling each other by an $h$-independent interaction. The equilibrium structure then necessarily must be equidistant spacing around the resulting ring for all particle numbers. The chain structure has disappeared. However, this structure is only reached in the extreme limit of $h=0$, while the chain structure is maintained for any finite, even infinitesimally small, value of $h / R$. The present calculations assume equidistant angular spacing, which implies that the in-chain particles approach each other in head-to-tail attractive configurations, and simultaneously all out-of-chain interactions become repulsive.
Then the energy scaling of these rather unstable configurations approach $h^{-3}$.

The understanding of equilibrium configurations as effective chain structures has some interesting consequences for the spectrum of normal modes.

\section{NORMAL MODES}

Vibrations around equilibrium configurations reveal intrinsic properties of the vibrating structures. The corresponding uncoupled motion of orthogonal modes can be found by expansion of the energy in Eq. (4) to second order in coordinate deviations from the set of their equilibrium values $\left\{\phi_{j}^{(0)}\right\}$. Then we have

$$
\begin{aligned}
E-E_{0} & \approx \frac{1}{2} \sum_{i, j} K_{i, j}\left(\phi_{i}-\phi_{i}^{(0)}\right)\left(\phi_{j}-\phi_{j}^{(0)}\right) \\
K_{i, j} & =\frac{\partial^{2} E}{\partial \phi_{i} \partial \phi_{j}}
\end{aligned}
$$

where $E_{0}$ is the energy of the equilibrium configuration. Both the vanishing (omitted) first and the second derivatives $(K)$ are taken at the equilibrium. The variable change corresponding to diagonalizing the matrix $K$ in Eq.(6) provides a set of eigenvalues defined by $\frac{1}{2} m \omega_{n}^{2}$. The resulting frequencies $\omega_{n}^{2}$ are all positive if the equilibrium configuration is stable. Conversely, if one or more eigenvalues, $\omega_{n}^{2}$, are negative, then the system is unstable towards coordinate deviations in the corresponding directions.

\section{A. Stability}

We calculate the elements of the $K$-matrix in Eq.(15) analytically from the expression in Eq.(3). We subsequently diagonalize this matrix and obtain the eigenvalues expressed as eigenfrequencies. The corresponding eigenvectors include all the $N$ angular coordinates, in contrast to the energy calculations in the previous section where constant differences between neighbouring particles were kept. This extension of configuration space implies that the computed eigenfrequencies can show instability corresponding to vibrational modes different from vibrations where the nearest neighbour distances are kept.

We emphasize that a near (first order) miss of the minimum due to a too restricted (constant difference) variational space do not jeopardize the stability condition since the second derivatives remain unchanged by moving from approximate to true (close-lying) minimum. A conclusion of stability (all $\omega_{n}^{2}$ are positive) of the calculated restricted solution then means that the marginally deviating true solution also is stable. On the other hand, instability of the restricted solution implies that the true solution may deviate substantially. 


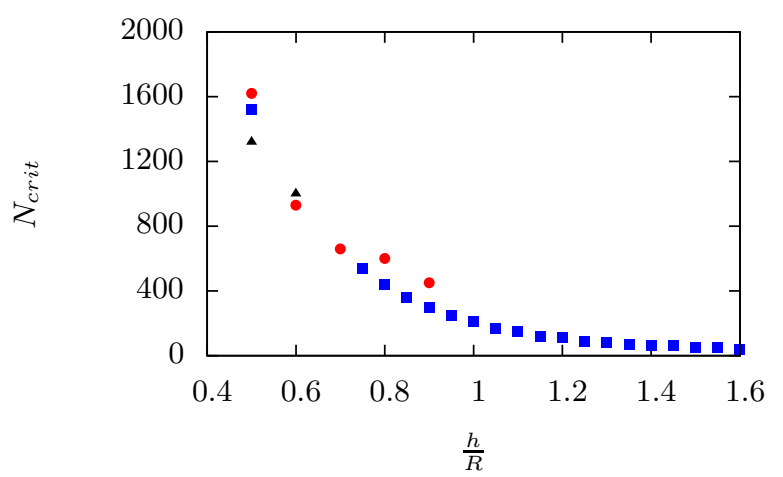

FIG. 6: The critical number of dipoles $N_{\text {crit }}$ as a function of $\frac{h}{R}$. For $N>N_{\text {crit }}$ the equidistant configuration becomes unstable. The blue square is for two dipoles per winding, the red circle is with three dipoles per winding and the black triangle is for 4 dipoles per winding

It turns out that the restricted configurations (constant angular separation) are not always stable, and that the stability depends, on both the geometry of the helix $\left(\frac{h}{R}\right)$, and the total number of dipoles $N$. The configuration with one dipole per winding is always stable as long as the short range interaction is repulsive, that is $\frac{h}{R}<\pi \sqrt{2}$. For two, three and more dipoles per winding the picture gets more complicated.

It is tempting to believe that distances corresponding to the minima in fig. 4 are good candidates for stable configurations. We therefore place the dipoles at these points with equidistant separation such that two, three and four dipoles are at $\phi \approx \pi, \phi \approx 2 \pi / 3$, and $\phi \approx \pi / 2$, respectively. We then increase the total number of particles, $N$, or equivalently the extension of the helix, and observe when, or possibly if, these configurations become unstable. Obviously, an unstable configuration must require another structure of lower energy. However, finding those structures for increasing $N$ quickly becomes a complicated many-body problem. These solutions are in general beyond the scope of this paper.

In fig. 6, the critical number of dipoles $N_{\text {crit }}$, is shown as a function of $\frac{h}{R}$. Values of $N$ smaller (larger) than $N_{\text {crit }}$ are then stable (unstable). If there is no point for a particular value of $\frac{h}{R}$, it is because the system is stable up to at least our maximum total number, $N=1600$. Two dipoles per winding are stable in the equidistant configuration when $\frac{h}{R}<0.65$, at least up to $N=1600$. For $\frac{h}{R}>0.65$ the system becomes unstable at much smaller values of $N$ decreasing from about 550 towards zero as $\frac{h}{R}$ increases. In contrast, for three dipoles per winding the critical value of $N$ decreases from about 1600 to about 500 when $\frac{h}{R}$ increases from 0.5 to 0.9 . For $\frac{h}{R}>0.9$, the equidistant configuration is stable for three dipoles per winding for all $N<1200$.

The stability behaviour for three and four dipoles per winding is similar but stability arises already for larger values than $h / R \approx 0.6$. This smaller threshold of $h$ -

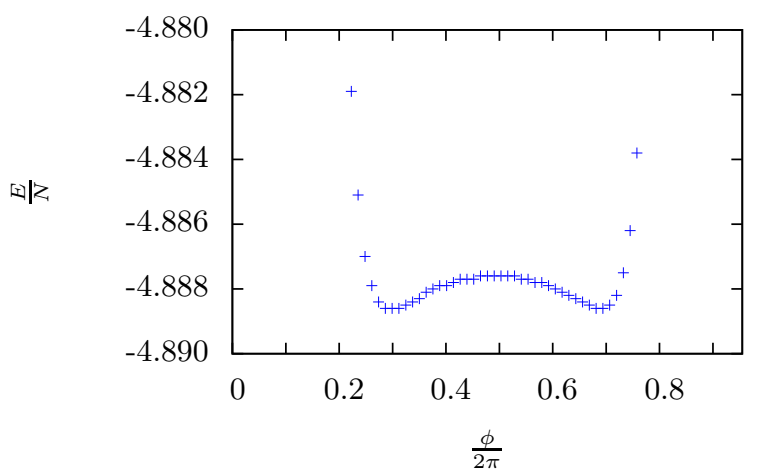

FIG. 7: The reduced energy per dipole, $E / N$, as function of the angular distance between two chains of dipoles for $N=$ 500 and $h=R$.

instability has to arise from the presence of the fourth particle. Roughly in the interval, $0.2<h / R<0.6$, the four equidistantly placed dipoles become unstable only for relatively large dipole number as shown in fig. 6. For small $h / R$-values, the behavior is similar to our three cases in fig. 6. The apparent extra stability of four chains are due to a strong repulsion between neighboring dipoles in the same circle. If possible, they lock each other in the minima seen in fig. 4.

When $h / R$ decreases below about 0.2 the equidistant four-particle system again becomes stable, but now due to the overwhelmingly dominating in-chain head-to-tail attraction. This configuration is reached by decreasing $h$ towards zero for equidistant angular distances. The stability in at least a local minimum is guaranteed for any particle number per winding by strong in-chain attraction and a comparably much smaller out-of-chain repulsion. The stability behavior for different dipole numbers is a sobering illustration of the possible many-body complexity of even the present one-dimensional system. The detailed structure-changes responsible for these instabilities are investigated in the next subsection.

\section{B. Chain structure}

To understand the computed properties we first return to the two chain structure illustrated in fig. 1 for two dipoles per winding. We focus on two nearest neighbour dipoles belonging to different chains. When $\frac{h}{R}$ increases for given $N$ their interaction changes from repulsive to attractive, and therefore it becomes energetically unfavourable to maintain the configuration of equal angular spacing. It is advantageoues to move the two chains closer to each other. For example, for $N \approx 600$ the stable configuration for small $\frac{h}{R}$ becomes unstable when $\frac{h}{R}$ increases above 0.75 .

This result can be understood by extending the helix by adding one pair at a time. Each of these extra dipoles at the end of the helix repel the neighbor in the oppo- 


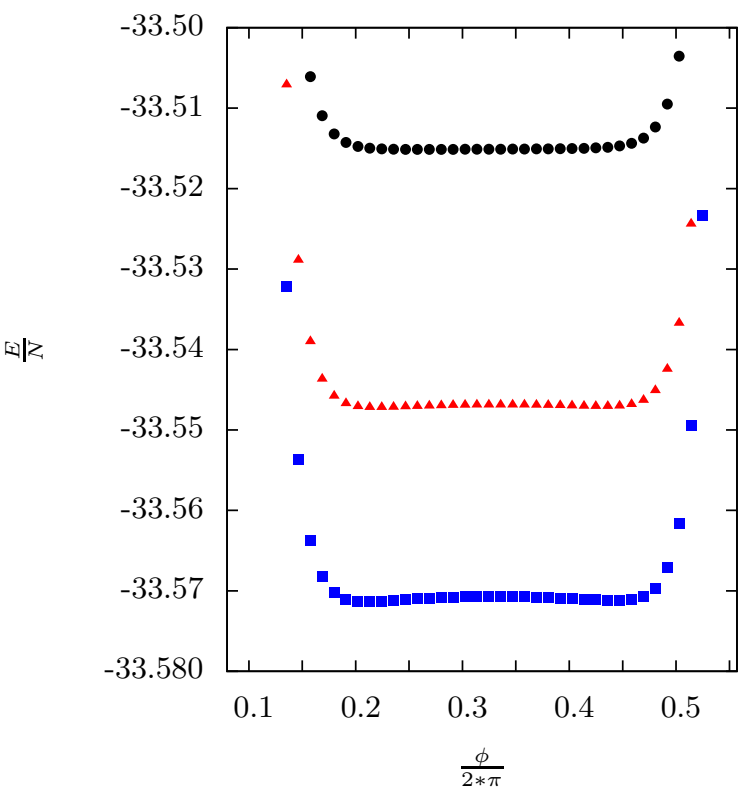

FIG. 8: Reduced energy per dipole, $\frac{E}{N}$, as a function of the position of a third chain of dipoles between two other chains, the first two chains are separated by $\phi=\frac{2}{3} 2 \pi$ radians, and the position of the third is measured from the first. This is done from top to bottom for $N=500$ (black circle) $N=700$ (red triangle) and $N=1000$ (blue square) dipoles and $\frac{h}{R}=0.6$.

site chain in the circle beneath. In contrast, the interaction to dipoles on circles far away would be attractive because such pairs approach the head-to-tail attractive configurations. The larger $N$ the more of these attractions which eventually would dominate over the repulsion between nearest neighbors. This is directly related to the two-body potential shown in fig. 3 where the attraction appears already for $\phi>2 \pi$.

This explanation is tested by varying the distance between the two chains in fig. 1 The resulting energy per dipole is shown in fig. 7, where the equidistant configuration corresponds to the angle close to $\pi$. The corresponding maximum demonstrate instability and the two minima at about 1.8 and 4.3 strongly suggest an equilibrium configuration where the two chains remain but at a smaller distance. The two minima are equivalent as each chain can be shifted by moving points either up or down the helix. A further confirmation of this two-chain interpretation for equidistant chains is derived from the fact that the first unstable normal mode with the imaginary frequency corresponds to the structure of two chains vibrating against each other. All other normal modes of equidistant chain vibrations reveal stability. The calculated normal mode frequencies for the new nonequidistant minimum configurations all show stability for large $N$.

We continue to test the idea of chains as the basic ingredients and explanations of the stability results in fig. [6 where three chains equidistantly placed on the helix becomes unstable for $N>N_{\text {crit }} \approx 900$ and $\frac{h}{R}=0.6$. The properties of this instability can be investigated by a procedure similar to that leading to fig. 7 . We first form two chains with an angular distance of $\phi=\frac{2}{3} 2 \pi$ corresponding to the third minimum in fig. [4 If a third chain of dipoles is placed directly in between the first two we recover the equidistant configuration for three dipoles per winding. Instead we vary the position of the third chain between the first two chains.

The resulting energies are shown in fig. 8 for three different total number of particles. For $N=500$ we see the minimum at $\phi=\frac{2}{3} 2 \pi$, that is directly between the two first chains corresponding to the equidistant configuration, and consistent with $N<N_{\text {crit }} \approx 900$. For $N=700$ it is difficult to extract an accurate value for the position of the minimum, but increasing further to $N=1000$ two separate minima appear on opposite site of the value for the (now unstable) equidistant configuration.

The interpretation is again supported by the observation that the structure of the first unstable normal mode with imaginary frequency corresponds to the two nearest chains vibrating against each other, while all other normal modes are stable. All these properties show that even when the equidistant configuration becomes unstable, the chain structure can still be considered the basic constituent. This means that the attraction of two dipoles above each other in a chain dominates over the interaction between dipoles in different chains.

\section{Small systems}

The long-distance interaction is responsible for many properties. In particular, it provides the attraction between particles on windings far apart leading to collapse of the system for sufficiently large $N$. By the same token small $N$ leads to stable systems with equidistant spacing of the dipoles for moderate values of $h / R$, see fig. 6. In addition small $N$ allow faster computations. We can exploit this fact to study generic properties like structures of low-lying normal modes.

We choose $N=12$ as this number of dipoles is divisible by both 2,3 and 4 dipoles per winding. This match is expected to provide more binding and very clean vibrational normal modes. We show the lowest frequencies in fig. 9 for 2,3 and 4 dipoles per winding. It is striking that the spectra are almost two-, three- and fourfold degenerate. This is caused by the commensurability to the number of particles per winding and implies that the eigenvalues appear either pairwise, as triples or as quadruplets. The degeneracies are getting less pronounced as the frequency increase.

These properties are signals of the chain structures discussed in the previous subsections. The computed degenerate normal modes correspond for two dipoles per winding to the two chains moving either exactly in phase preserving the same particle distances, or chains moving against each other exactly in opposite phase. In general, 
Eigen mode
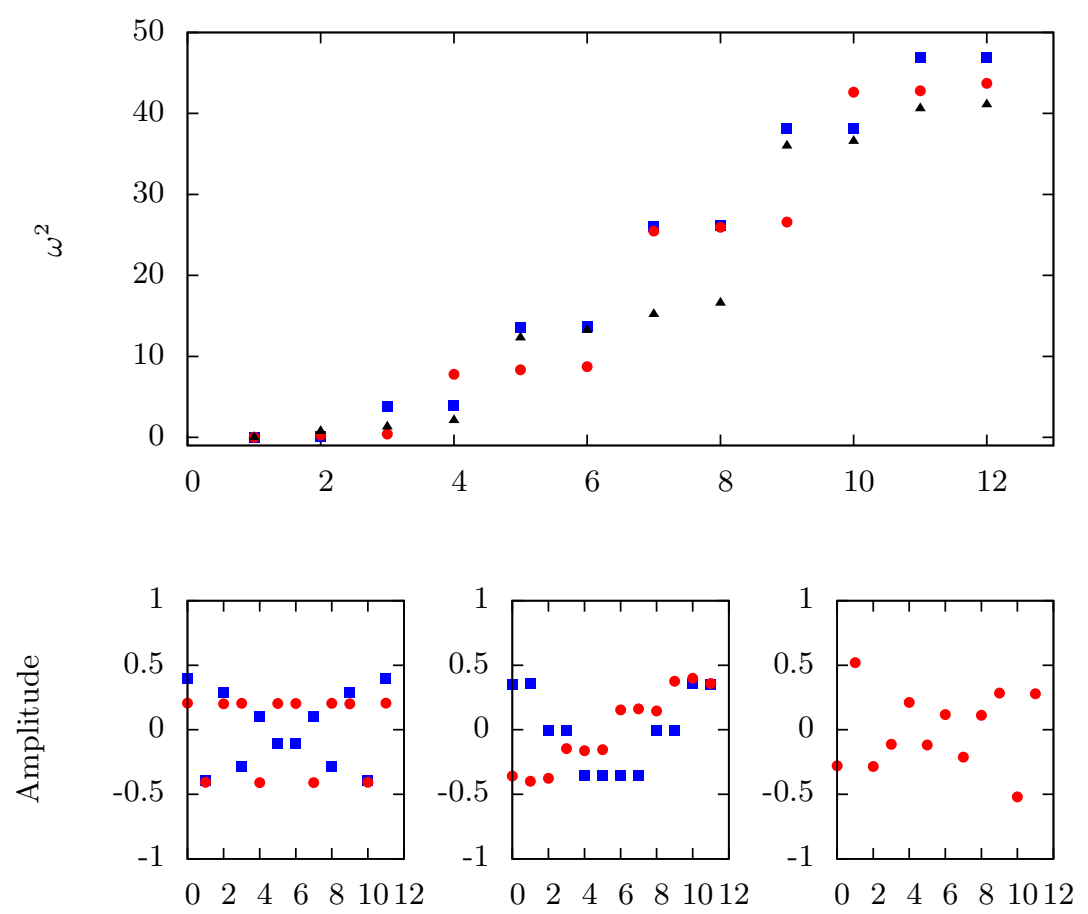

Dipole number along the helix

FIG. 9: (top) The spectrum of squared frequencies for 12 dipoles, with two (blue square ), three (red circle) and four (black triangle) dipoles per revolution. (bottom) The amplitudes of the normal modes for the lowest nearly degenerate eigenvalues. Left is eigenmode number 3 (blue square) and 4 (red circle), repectively for two and three dipoles per windings. Middle is eigenmode number 4 (blue square) and 5 (red circle), repectively for two and three dipoles per windings. Right is eigenmode number 6 (red circle) for three dipoles per windings.

we characterize each of the normal mode oscillations by a set of relative amplitudes, where each describes the maximum deviation from equilibrium along the helix of the corresponding dipole. The amplitudes of these oscillations are also shown in fig. 9. The same degeneracies at higher frequencies correspond to the same vibrational relation between chains but now with additional nodes along the chains.

For two particles per winding the amplitudes for eigenmode number 3 (bottom left in fig. 9) show alternating direction for neighboring dipoles. This means that the two chains oscillate against each other. For three particles per winding the eigenmodes number 4,5,6 are shown from left to right in bottom panel of fig. 9. Left show two chains moving against the third as seen by two almost equal neighboring amplitudes and one amplitude of about twice the size in opposite direction. Similarly, in the middle are the three chains in phase as the three neighboring amplitudes are equal. On the right we see again two chains moving against the third.

For four particles per winding (not shown on figures) the four normal modes are similarly related to chains moving in phase, or pairs moving against each other, or triples against a single chain. The degeneracies and the normal mode structure are effects of the next-nearest neighbour interaction, and the small difference within the almost degenerate frequencies are due to small nearestneighbour corrections to the vibration-dominating nextnearest interaction.

These simple structures arise so purely because an integer number of particles can precisely fit into one winding. If this is not the case the degeneracies are broken and the spectra would move towards another type of degeneracy. A non-integer ratio between particle number and particles per winding must destroy the picture of chain structures, simply because identical chains cannot be formed. However, the lowest energy from the strongest attraction arises by adding the odd particles at the end of the chains. These features are illustrated by the example of $N=13$ shown in fig. 10, where top panel exhibits frequencies for 2,3 or 4 dipoles per winding with the additional dipole placed at the end of one of the chains.

The degeneracies compared to $\mathrm{N}=12$ are now lifted although traces can be detected. However, the modes exhibited in fig. 9 still come in pairs for two dipoles per winding, even though their frequencies differ. The same features of normal mode similarity between systems with $N=12$ and 13 are also seen for the energies of three and four particles per winding. The amplitudes for two modes of two dipoles per winding at the bottom of fig. 9 
show that one of the chains essentially is not moving while the other is undergoing an odd parity oscillation. The interpretation is in general that the slightly different chains still vibrate against each other. These boundary layer effects would decrease with increasing total number of particles.

\section{SPEED OF SOUND}

The speed of sound in a crystal is a fundamental property which plays an important role in many physical processes. The sound is mediated by particle motion and normal modes carry the necessary information about the correlated small amplitude vibrations which form the sound waves. The length of our one-dimensional regular crystal is $N R(\Delta \phi)_{0}$, where $(\Delta \phi)_{0}=\phi_{i+1}-\phi_{i}$ is the distance between equally spaced neighbouring dipoles along the helix. The corresponding wave numbers are $k_{n}=2 \pi n /\left(N R(\Delta \phi)_{0}\right)$ where $n$ is an integer. The speed of sound is then given by $c=\omega_{n} / k_{n}$ in the limit of small $k_{n}$ when $\omega_{n}$ is the n'th normal mode frequency. Since small $k_{n}$ requires large $N$ we have to find normal modes for large systems.

\section{A. Simple models}

The restoring force is in general obtained from the energy in Eq. (5) for arbitrary, independent displacements of the individual dipoles. The stable configurations are equidistantly distributed dipoles along the helix for a modest density per revolution and perhaps also only for a finite total number of particles. For this structure we search for small amplitude vibrations around equilibrium which allows sufficiently accurate energy calculation by using second order as in Eq. (5). Large $N$ is expected to eliminate dependence on specific choices of end-point configurations. We shall explore consequences of these approximations while staying within the validity ranges found in the previous sections.

The equal spacing condition for a very long sequence of dipoles has several consequences we must take into account. First, the same translation of all dipoles does not change any of the relative distances, and the total energy must remain unchanged as well. Second, all two-body properties only depend on the relative distance between the particles. In particular, the value of $K_{i j}$ is then the same as $K_{k+i, k+j}$ for any value of $k$. The double sum in Eq. (5D) can then be performed in different order where the summation indices are along constant sum, $k$, and constant difference, $l$. However, only relative distances produce any energy variation. Let us measure deviations from equilibrium positions for the individual variables, that is using the coordinates $\tilde{\phi}_{i}=\phi_{i}-\phi_{i}^{(0)}$. In addition, we assume that the energy to second order in distances from equilibrium is independent of which particle is used as the reference position. These assumptions lead to

$$
E-E_{0}=\sum_{l>0} \frac{1}{2} K_{k+l, k-l}\left(\tilde{\phi}_{k+l}-\tilde{\phi}_{k}\right)\left(\tilde{\phi}_{k-l}-\tilde{\phi}_{k}\right),
$$

where we measure relative to particle $k$. Eq.(7) is then independent of $k$ and the curvature only depends on $l$.

The classical force on particle $n$ is minus the gradient of the potential energy given in Eq. (77) which according to Newton's second law equals mass times acceleration given as the second time derivative. The equation of motion becomes

$$
m \frac{\partial^{2} \tilde{\phi}_{k}}{\partial t^{2}}=\frac{1}{2} \sum_{l>0} K_{l}\left(\tilde{\phi}_{k+l}+\tilde{\phi}_{k-l}-2 \tilde{\phi}_{k}\right)
$$

where we used the definitions $K_{l}=K_{k+l, k-l}$. We now search for periodic motion, $\tilde{\phi}_{j}=A_{j} \cos \left(\omega t+a_{j}\right)$, around the $j$ equilibrium positions. The time dependence cancel on both sides of Eq. (8) if and only if the amplitudes are identical, that is $A_{j}=A_{0}$, and simultaneously the average phase $\left(a_{k-l}+a_{k+l}\right) / 2$ equals $a_{k}$ for all $l$. The latter condition immediately implies a linear dependence of the phases, that is $a_{j}=b_{0}+j b_{1}$. With these definitions we arrive at the frequency given by

$$
\begin{aligned}
m \omega^{2} & =\sum_{l>0} K_{l}\left(1-\cos \left(l b_{1}\right)\right) \\
& =2 \sum_{l>0} K_{l} \sin ^{2}\left(l b_{1} / 2\right) .
\end{aligned}
$$

These frequencies with the corresponding eigen phases given by $b_{1}$ characterize the $N$ independent periodic solutions. The spectrum is continuous as any value of $b_{1}$ between 0 and $2 \pi$ provide a frequency through Eq. (9). An appropriate discretization can for example be found by assuming the same phase, apart from a multiplum of $2 \pi$, for the end-point dipoles. This means that $a_{0}=a_{N}+n 2 \pi$ which is equivalent to $b_{1}=2 \pi n / N$ for any integer value $n$. This could be obtained by bending the helix to let the two end-points meet. Now the $N$ independent solutions, $\omega_{n}$, result from Eq. (9).

The size of $K_{l}$ decreases strongly with $l$, since the overall energy scale is obtained from a cubic decrease with distance. The largest $K_{l}$ is then usually by far the lowest $l$, that is $l=1$. By using only lowest order in an expansion of Eq. (9), we find the lowest frequencies to be approximately given by

$$
\omega_{n} \approx \frac{2 \pi n}{N} \sqrt{\sum_{l>0} l^{2} K_{l} / m} .
$$

The speed of sound, $c$, is then obtained as the ratio between $\omega_{n}$ and the wave number $k_{n}$, where the latter is found by equating the total length of the helix with the wave length. Thus, $R N(\Delta \phi)_{0}=2 \pi n / k_{n}$, where $(\Delta \phi)_{0}$ is the angular distance between the equidistantly placed 
Eigen mode
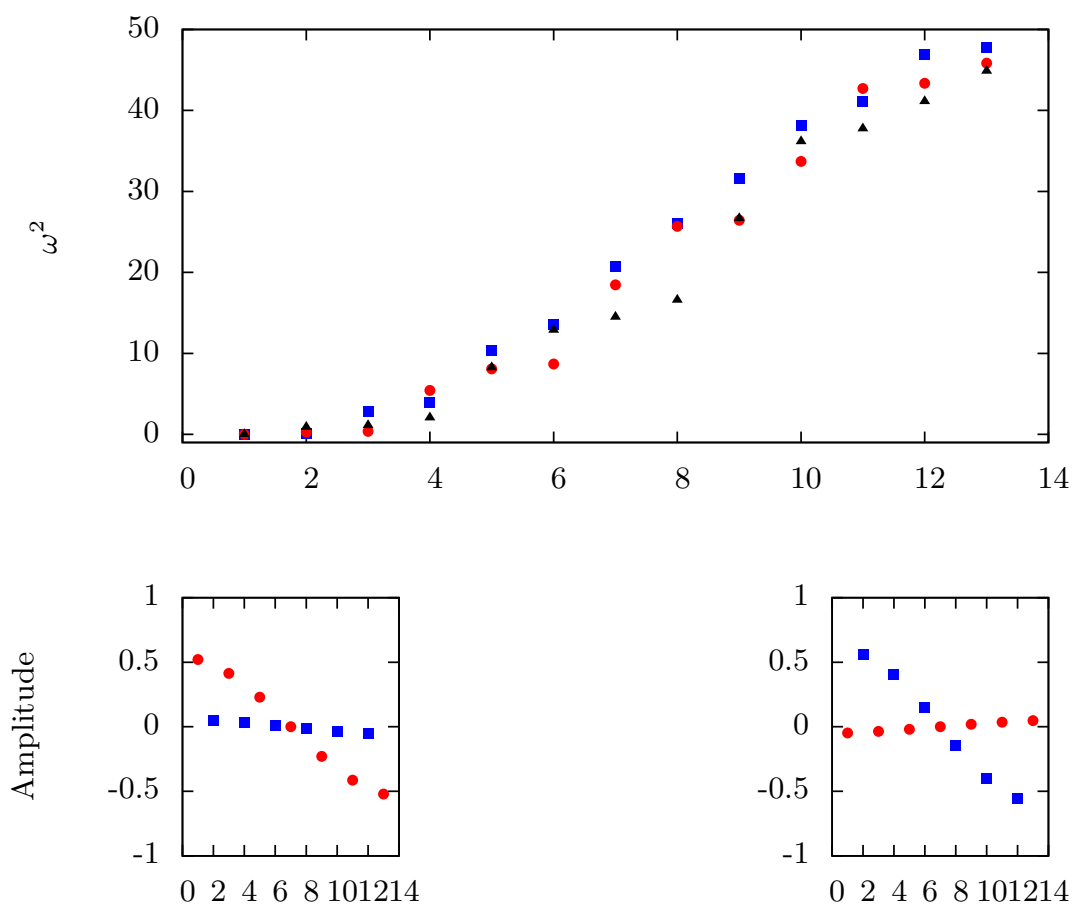

Dipole number along the helix

FIG. 10: (top) The spectrum of squared frequencies for 13 dipoles, with two (blue square ), three (red circle) and four (black triangle) dipoles per revolution. (bottom) The amplitudes of the normal modes for the lowest nearly degenerate eigenvalues of two dipoles per winding. Left is eigenmode number 3 where the long chain (red circle) is moving while the short chain (blue square) remains at rest. Right is eigenmode number 4 where the cjhains exchange motion.

dipoles. We then finally get

$$
c=\frac{\omega_{n}}{k_{n}} \approx R(\Delta \phi)_{0} \sqrt{\sum_{l>0} l^{2} K_{l} / m},
$$

which is the result for a periodic structure of particles located on a ring [17]. Thus we get the same $c$ independent of which of the lowest modes of $n=1,2,3$ we used. Furthermore, $c$ is also roughly independent of the number of dipoles per winding. This is seen from Eq. (9) when the $K_{l}$-summation is dominated by the first in-chain contribution for $l=l_{0}$ giving $c=R(\Delta \phi)_{0} l_{0} \sqrt{K_{l_{0}} / m}$. We then obtain the same $c$, since both $K_{l_{0}}$ and $(\Delta \phi)_{0} l_{0}$ are independent of $l_{0}$ or equivalently dipole density.

\section{B. Numerical results}

We calculate all $N$ eigenfrequencies, $\omega_{n}$, as functions of $N$ for 1,2,3,4 dipoles per revolution in the equidistant stable configurations. We first show the ordered frequencies for $N=20,50,100$ and $h=R$ in fig. 11. The $x$-axis is scaled with the different number of dipoles. The details are not meant to be visible on this figure, where the striking features are that all eigenfrequencies, after scal-

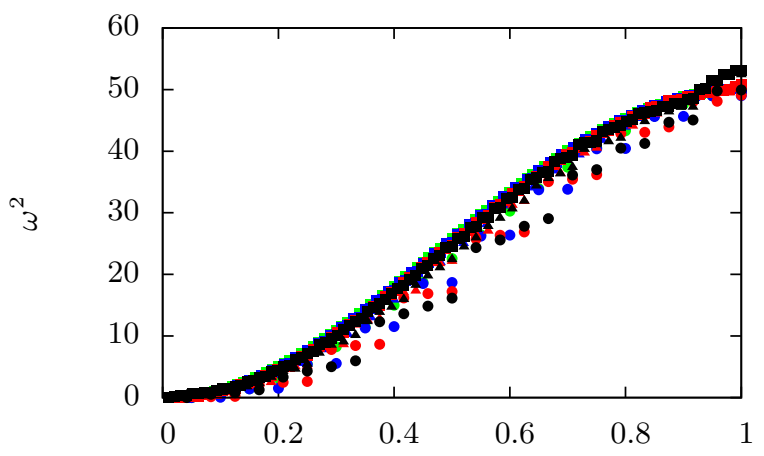

FIG. 11: The squared eigenfrequencies with $h / R=1$ for $N=20$ (circle), 50 (triangle), and 100 (square) as functions of their number in the ordered sets of eigenvalues divided by the total number of dipoles. The dipoles per revolution are 1 (green), 2 (blue), 3 (red), and 4 (black).

ing, essentially follow the same curve varying from zero to a maximum value of about 50 for the largest frequency.

This behaviour is evident from Eq. (9) when the discretization of $b_{1}$ is considered to vary from 0 to $2 \pi$. These two end-point values of $b_{1}$ result in the maximum value of the cosine function, and a limiting largest value obtained 


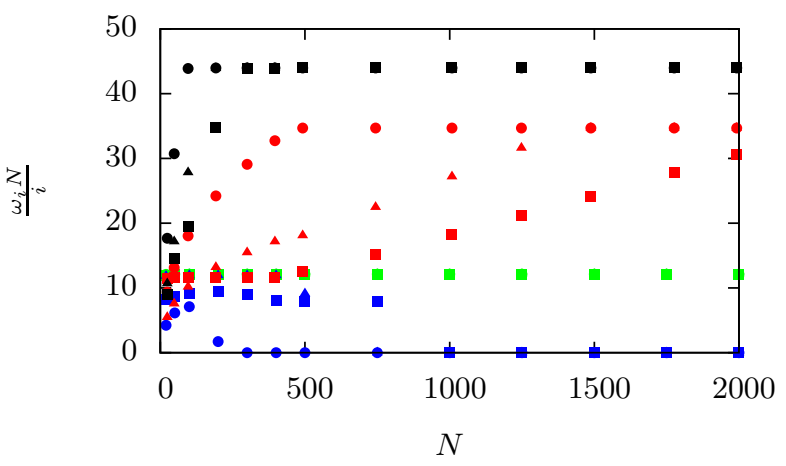

FIG. 12: The lowest three eigenfrequencies multiplied by $N$ and divided by respectively 1 (circle), 2 (triangle), and 3 (square) exhibited as functions of $N$. We choose $h=R$ and the number of dipoles per revolution to be 1 (green), 2 (blue), 3 (red), and 4 (black).

as the sum of all the curvatures. This sum is essentially independent of $N$, since $K_{l}$ decreases very fast with the distance between the dipoles as parametrized by the in$\operatorname{dex} l$. Thus, only the smallest values of $l$ then contribute in the summation. Values of $b_{1}$ around $\pi$ produce alternating signs of the cosine function and give the small frequencies.

We now focus on the lowest frequencies which according to Eq. (9) are expected to vanish as $n / N$ for increasing $N$. We therefore show the frequencies multiplied by $N / n$ in fig. 12 as functions of $N$ for the previous case of a few dipoles per revolution. The linear dependence is quickly reached for most of the stable systems. This arises from the allowed lowest order substitution of the argument in the sine function of Eq. (9). Here $2 \pi n / N \ll 1$ or equivalently $N$ must considerably exceed $6 n=6,12,18$, which easily should be fulfilled for our cases. Also the increase proportional to $n$ is seen in all cases.

An exception in fig. 12 is seen for two dipoles per revolution where the small $N$ increase is followed by a maximum and a subsequent decrease. This behaviour reflects the transition towards instability of the configuration of equidistant spacing. Another exception appears for three particles per revolution. The constant values approached by the second and third lowest frequencies occur apparently for much larger values of $N$, that is about 500 and 1500. The latter anomaly can be explained by starting with very small $N$ where we know that a near threefold degeneracy appear. Increasing $N$ the two lowest frequencies increase, whereas the third stabilizes and remain at this constant value when $N$ passes 500 . The other two, initially lowest, frequencies continue to increase until about 1500 where a new stabilization takes place due to the crossing of an initially much higher lying mode.

The stability of $N \omega_{n}$ for large $N$ reflect the appearance of acoustic modes which, as we shall discuss now, corresponds to in-chain dipole oscillations. The frequency

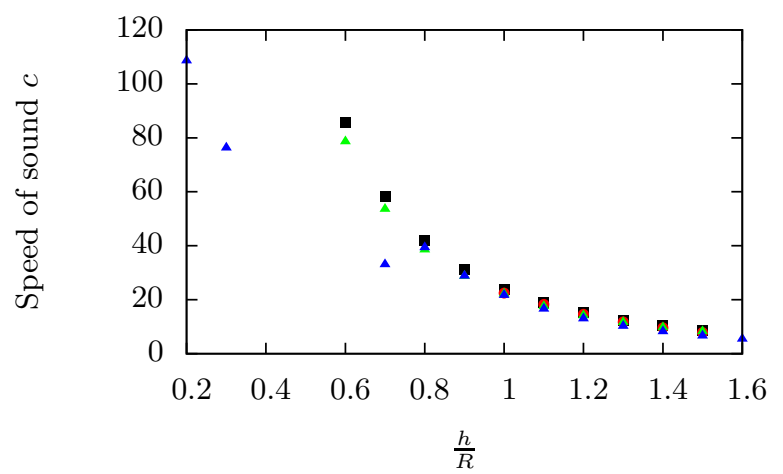

FIG. 13: The Speed of sound c obtained for $n=1$ as a function of the ratio $\frac{h}{R}$ for one dipole per revolution (black square), three dipoles per revolution (red circle), four dipoles per revolution (blue nabla) and a nearest-neighbour calculation for 1 dipole (green triangle)

crossings of these modes by lower-lying modes for small $N$ reflect that out-of-chain oscillations are more favorable for small chains.

The features discussed here are exemplified with $h=R$ results. However, the general behaviour for other helix parameters can be understood with the same ingredients. The curvatures would change and accordingly also the frequencies. The only other differences are that the stabilities depend rather strongly on $h / R$ and the large $N$-limit and the resulting linear dependence on $1 / N$ may sometimes not be reached for the equidistant configurations.

We now proceed to calculate the speed of sound by dividing the frequencies by the wave number as in eq. (11). This means multiplying the numbers in fig. 12 with $\left.R(\Delta \phi)_{0}\right) /(2 \pi n)$ where $n$ is 1,2 and 3 . The results are shown in fig. 13 for $n=1$ as function of $h / R$. A smooth curve for one dipole per revolution is found for a large range of helix parameters. The speed of sound decreases roughly as $(R / h)^{3}$ with increasing $h / R$. This is almost the same scaling as observed for the energy, see fig. 5 . These results are calculated from the lowest frequency but essentially the same curve appears by use of the second and third frequencies corresponding to $n=2,3$. The proportionality in $n$ from Eq. (11) is cancelled by that in $k_{n}$.

More dipoles per revolution also lead to series of points in fig. 5. However, instability at large $N$ before stabilization prevents calculation of the speed of sound and fewer points can be obtained. It is striking that all available points appear essentially on the same curve as the onechain result. The equidistant equilibrium spacing, $(\Delta \phi)_{0}$, decreases with the number of dipoles per revolution. The factor of $l^{2}$ inside the square-root in Eq. (11) can compensate this when a single value of $K_{l}$ dominates the speed of sound. This happens in the chain picture. Here $K_{l}$ will be small for $l$ less than the number of chains, $l_{0}$. However, $K_{l_{0}}$ will be the term describing the interaction 
with the nearest-neighbour within a single chain. The $K_{l}$ terms for $l<l_{0}$ are interactions with particles in different chains. In summary, we have a chain dominated dynamics where we can use an in-chain nearerst-neighbour approximation for any number of chains. Thus, the sound waves travel within each chain, and the perturbations of the waves within a single chain from neighbouring chains are minimal.

Finally, we investigate the consequences of the instability of some of the configurations. For $h=R$ we know that the chains prefer specific distances for sufficiently large $N$ as exemplified in figs. 7 and 8 . We therefore calculate the normal mode spectra for the non-equidistant equilibrium configurations of two dipoles per winding. The resulting points for an average value, $(\Delta \phi)_{0}$, fall on the common curve in fig. 13 found for the equidistant configurations. This again support the interpretation in terms of chain structures given above.

\section{DISCUSSION AND OUTLOOK}

The classical potential energy is calculated for a total large number, $N$, of dipoles placed on a helix and uniformly distributed with a density corresponding to 2,3 and 4 dipoles per winding. The dependence on the ratio of the two helix lengths, pitch-to-radius $(h / R)$, is investigated. The unit of energy is given by dipole moment squared divided by the cubic power of the radius. These parameters only enter as a scale without influence on the geometric structures providing stability. The one dimensional confinement implies that the dipoles prefer to be located at specific angular distances from each other along the helix. This is due to the strong attraction between head-to-tail configurations combined with equally strong side-by-side repulsion. The system collapses into strings of infinite energy when $h>R \pi \sqrt{2}$, which therefore is a limit we do not exceed.

The stable structures for relatively small densities can be characterized as a number of chains formed by dipoles almost head-to-tail along the helix. The stable chain structures are equidistantly distributed when $h / R$ is larger than about 1 and $N$ is relatively small. Increasing $N$ for these $h / R$ values leads to instability. For decreasing $h / R$ stability is maintained for larger $N$ but eventually lost for all densities. The structures with lower energy that cause this instability in the case with a few dipoles per winding are clusterized states where chains of dipoles cluster. These modes of instability are investigated through the classical normal modes. An abrupt change of the chain structures occurs when $h / R$ is only marginally varied. This is analogous to spontaneou symmetry breaking of a symmetric minimum by conversion into a maximum and two asymmetric minima. A main finding is that both initial and final structures can be characterized as chains and thus the change is really in the local density due to clusterization.

By increasing the density the preferred configuration changes from one to more chains. For densities correponding to less than five dipoles per winding the equidistant spacing around the circles are broken for a sufficiently extended helix. The more chains imposed, the more stability because the short-range repulsion between neighboring dipoles on the circles lock each other in positions on average as far away as possible. This is classical crystal formation.

Detailed investigations are also carried out for smaller $N$ where we find signals of preferred geometries in degeneracies, or lack of them, in the normal mode frquenies. For example $N=12$ allow very symmetric structures of 2, 3 and 4 chains with corresponding normal mode degeneracies of 2, 3 and 4 . The normal mode amplitudes reflect preference for chains as inert units vibrating against each other. The degeneracies are broken by addition of one more dipole at the end of one chain. However, the chains still prefer to vibrate in the same way as inert entities. Finally we calculate the speed of sound whenever we find stable structures for sufficiently large $N$. We find the same behaviour of the speed of sound as function of $h / R$ for 2,3 and 4 chains in the system. This confirms the chain picture as it shows that sound waves propagate along the chains.

In this paper we investigated a finite number of dipoles on a finite helix. Boundary conditions corresponding to densities of a non-integer average number of dipoles per winding were only discussed for a few small systems. For larger systems the optimum configurations would be chains found by dividing the dipole number by the number of windings and rounding up to the nearest interger. This leaves room for adding the non-matching dipoles at the outer circle. They would each prefer to be located in continuation of one of the chains. For very large systems these end-point structures are not expected to influence the bulk structures.

In future studies, we want to consider the quantum effects on the system both from the few-body and the many-body point of view. For few-body quantum bound states, previous studies have considered dipolar particles in one-dimensional tubes and on rings [36 40]. Dipolar interactions can be addressed using harmonic approximations [1] 43], stochastic variational methods [44], and exact diagonalization [45 47]. All of these methods should be adaptable to the helical geometry. For treatment of larger particle numbers, we imagine that a combination of the Luttinger approaches with dipolar particles 28, [8 54], and matrix product states $[55]$ or density matrix renormalization group methods [56, 57] are possible approaches. Some recent examples of the numerical methods applied to dipolar particles can be found in Refs. 58 61.

As discussed briefly in the introduction, we expect that the most straighforward experimental realization of helical geometries will be in systems where light is guided by an optical nanofiber with a diameter that is smaller than the wave length of the light such that an evanescent wave builds that can be used as an atomic trap This 
has been done by the Rauschenbeutel group in Vienna [25 27]. The same group has suggested the creation of helical traps in a recent paper [15]. At the moment these proposals involve trapping of non-polar Rubidium atoms and thus one would need to extend the technique to either polar molecules or to atoms with large intrinsic magnetic dipolar moments. Given the added complications in producing cold polar molecules, it may be more easy to use magnetic dipoles.

Our study here concerns a single helix. An obvious extension is to the double helix DNA geometry proposed in Ref. [15]. Based on the current finding one might expect that this would merely be a doubled version of the single helix ground states. However, due to the long-range interactions one could imagine that more involved states would be allowed. In terms of quantum mechanical few- and many-body states this should be directly addressible using the techniques discussed above. An interesting recent proposal concerns a generalization to a system where there are three helical traps intertwined corresponding to the geometry of a three-stranded DNA molecules. In this case it has been suggested that three-body bound states across the strands could occur in the case where no two-body bound states across any two out of the three strands are possible 62, 63. This could connect such systems to the Efimov physics studied in few-body nuclear and atomic physics [64]. Again the methods mentioned above would be able to address such ideas.

The authors acknowledge several inspiring conversations with G. De Chiara and support from the Danish Council for Independent Research.
[1] Ospelkaus S, Pe'er A, Ni K-K, Zirbel J J, Neyenhuis B, Kotochigova S, Julienne P S, Ye J and Jin D S 2008 Nature Phys. 4622

[2] Ni K-K, Ospelkaus S, de Miranda M H G, Pe'er A, Neyenhuis B, Zirbel J J, Kotochigova S, Julienne P S, Jin D S and Ye J 2008 Science 322231

[3] Deiglmayr J, Grochola A, Repp M, Mörtlbauer K, Glück C, Lange J, Dulieu O, Wester R and Weidemüller M 2008 Phys. Rev. Lett. 101133004

[4] Lang F, Winkler K, Strauss C, Grimm R and Hecker Denschlag J 2008 Phys. Rev. Lett. 101133005

[5] Ospelkaus S, Ni K-K, Wang D, de Miranda M H G, Neyenhuis B, Quéméner G, Julienne P S, Bohn J L, Jin D S and Ye J 2010 Science 101853

[6] Ni K-K, Ospelkaus S, Wang D, Quéméner G, Neyenhuis B, de Miranda M H G, Bohn J L, Ye J and Jin D S 2010 Nature (London) $\mathbf{4 6 4} 1324$

[7] Shuman E S, Berry J F and DeMille D 2010 Nature (London) 467820

[8] de Miranda M H G, Chotia A, Neyenhuis B, Wang D, Quemener G, Ospelkaus S, Bohn J L, Ye J and Jin D S 2011 Nature Phys. 7502

[9] Chotia A, Neyenhuis B, Moses S A, Yan B, Covey J P, Foss-Feig M, Rey A M, Jin D S and Ye J 2012 Phys. Rev. Lett. 108080405

[10] Baranov M A 2008 Phys. Rep. 46471

[11] Lahaye T, Menotti C, Santos L, Lewenstein M and Pfau T 2009 Rep. Prog. Phys. 72126401

[12] Carr L D, DeMille D, Krems R V and Ye J 2009 New J. Phys. 11055049

[13] Baranov M A, Dalmonte M, Pupillo G and Zoller P 2012 Chem. Rev. 1125012

[14] Lushnikov P M 2002 Phys. Rev. A 66051601

[15] Reitz D and Rauschenbeutel A 2012 Opt. Commun. 285 4705

[16] Giamarchi T 2003 Quantum Physics in One Dimension (Oxford University Press Inc., New York)

[17] Ashcroft N W and Mermin N D 1976 Solid State Physics (Saunders College, Philadelphia)

[18] MacDonald M P et al 2002 Opt. Commun. 20121

[19] Pang Y K et al 2005 Opt. Express 137615

[20] Bhattacharya M 2007 Opt. Commun. 279219
[21] Ricardez-Vargas I and Volke-Sepúlveda K 2010 J. Opt. Soc. Am. B 27948

[22] Okulov A 2012 Phys. Lett. A 376650

[23] Arnold A S 2012 Optics Lett. 372505

[24] Beattie S, Moulder S, Fletcher R J and Hadzibabic Z 2013 Phys. Rev. Lett. 110, 025301

[25] Sagué G, Baade A and Rauschenbeutel A 2008 New J. Phys. 10113008

[26] Vetsch E, Reitz D, Sagué G, Schmidt R, Dawkins S T and Rauschenbeutel A 2010 Phys. Rev. Lett. 104203603

[27] Dawkins S T, Mitsch R, Reitz D, Vetsch E and Rauschenbeutel A 2011 Phys. Rev. Lett. 107243601

[28] Law K T and Feldman D E 2008 Phys. Rev. Lett. 101 096401

[29] Astrakharchik G E, Morigi G, de Chiara G and Boronat J 2008 Phys. Rev. A 78063622

[30] Astrakharchik G E, de Chiara G, Morigi G and Boronat J 2009 J. Phys. B: At. Mol. Opt. Phys. 42154026

[31] Fishman S, de Chiara G, Calarco T and Morigi G 2008 Phys. Rev. B 77064111

[32] de Chiara G, del Campo A, Morigi G, Plenio M B and Retzker A 2010 New J. Phys. 12115003

[33] Huhtamäki J A M and Kuopanportti P 2010 Phys. Rev. A 82053616

[34] Schmelcher P 2011 Europhys. Lett. 9550005

[35] Zampetaki A V, Stockhofe J, Krönke S and Schmelcher P 2013 Phys. Rev. E 88043202

[36] Deuretzbacher F, Cremon J C and Reimann S M 2010 Phys. Rev. A 81 063616; Erratum: 2013 Phys. Rev. A $87039903(\mathrm{E})$

[37] Klawunn M, Duhme J and Santos L 2010 Phys. Rev. A 81013604

[38] Zöllner S, Bruun G M, Pethick C J and Reimann S M 2011 Phys. Rev. Lett. 107035301

[39] Zöllner S 2011 Phys. Rev. A 84063619

[40] Volosniev A G et al 2013 New J. Phys. 15043046

[41] Armstrong J R, Zinner N T, Fedorov D V and Jensen A S 2011 J. Phys. B: At. Mol. Opt. Phys. 44, 055303

[42] Armstrong J R, Zinner N T, Fedorov D V and Jensen A S 2012 Eur. Phys. J. D 66, 85

[43] Fil D V and Shevchenko S I 2014 arXiv:1401.7548

[44] Volosniev A G, Fedorov D V, Jensen A S and Zinner N 
T 2012 Phys. Rev. A 85023609

[45] Cremon J C, Bruun G M and Reimann S M 2010 Phys. Rev. Lett. 105255301

[46] Zinner N T et al. 2011 Phys. Rev. A 84063606

[47] Kristinsdóttir L H et al 2013 Phys. Rev. Lett. 110085303

[48] Citro R, Orignac E, De Palo S and Chiofalo M L 2007 Phys. Rev. A 75 051602(R)

[49] Citro R, De Palo S, Orignac E, Pedri P and Chiofalo M L 2008 New. J. Phys. 10045011

[50] Chang C-M, Shen W-C, Lai C-Y, Chen P and Wang D-W 2009 Phys. Rev. A 79053630

[51] Huang Y-P and Wang D-W 2009 Phys. Rev. A 80053610

[52] Dalmonte M, Pupillo G and Zoller P 2010 Phys. Rev. Lett. 105140401

[53] Kollath C, Meyer J S and Giamarchi T 2008 Phys. Rev. Lett. 100130403

[54] Lecheminant P and Nonne H 2012 Phys. Rev. B 85 195121
[55] Verstreate F, Murg V and Cirac J I 2008 Advances in Physics 57143

[56] White S R 1992 Phys. Rev. Lett. 692863

[57] Schollwöck U 2005 Rev. Mod. Phys. 77259

[58] Argüelles A and Santos L 2007 Phys. Rev. A 75053613

[59] Knap M, Berg E, Ganahl M and Demler E 2012 Phys. Rev. B 86064501

[60] Ruhman J, Dalla Torre E G, Huber S D and Altman E 2012 Phys. Rev. B 85125121

[61] Gammelmark S and Zinner N T 2013 Phys. Rev. B 88 245135

[62] Maji J, Bhattacharjee S M, Seno F and Trovato A 2010 New J. Phys. 12083057

[63] Pal T, Sadhukhan P and Bhattacharjee S M 2013 Phys. Rev. Lett. 110028105

[64] Zinner N T and Jensen A S 2013 J. Phys. G: Nucl. Part. Phys. 40053101 